\title{
Effect of ion bombardment on in-plane texture, surface morphology, and microstructure of vapor deposited $\mathrm{Nb}$ thin films
}

\author{
Hong $\mathrm{Ji}^{\mathrm{a})}$ \\ Physics Department, University of Michigan, Ann Arbor, Michigan 48109
}

Gary S. Was

Department of Nuclear Engineering and Radiological Sciences, and Department of Materials Science and Engineering, University of Michigan, Ann Arbor, Michigan 48109

J. Wayne Jones

Department of Materials Science and Engineering, University of Michigan, Ann Arbor, Michigan 48109

Neville R. Moody

Sandia National Laboratories, Livermore, California 94551-0969

(Received 7 November 1996; accepted for publication 31 January 1997)

\begin{abstract}
Niobium films were deposited by physical vapor deposition (PVD) and ion-beam-assisted deposition (IBAD) using ion energies of $0,250,500$ and $1000 \mathrm{eV}$, and $R$ ratios (ion-to-atom arrival rate ratio) of $0,0.1$, and 0.4 on (100) silicon, amorphous glass, and (0001) sapphire substrates of thickness $50-1000 \mathrm{~nm}$. Besides a $\{110\}$ fiber texture, an in-plane texture was created by orienting the ion beam with respect to the substrate. The in-plane texture as measured by the degree of orientation was strongly dependent on both ion-beam energy and the $R$ ratio. In fact, the degree of orientation in the films followed a linear relationship with the energy per deposited atom, $E_{n}$. The grain structure was columnar and the column width increased with normalized energy. The surface morphology depended on both the normalized energy of the ion beam and the film thickness. All films had domelike surface features that were oriented along the ion-beam incident direction. The dimension of these features increased with normalized energy and film thickness. Surface roughness also increased with normalized energy and film thickness, with the root-mean-square roughness increasing from $1.6 \mathrm{~nm}$ for the PVD sample (100 nm thick) to $36.7 \mathrm{~nm}$ for the IBAD film (1000 eV, $R=0.4,800 \mathrm{~nm}$ thick). Both the surface morphology evolution and in-plane texture development in these films were the result of the different ion sputter rates among differently oriented grains.

(C) 1997 American Institute of Physics. [S0021-8979(97)09309-2]
\end{abstract}

\section{INTRODUCTION}

Texture in thin films has been found to greatly influence the performance of the materials in many applications such as microelectronics and metal/ceramic multilayer structural systems. In microelectronic applications, the different texture in aluminum thin films used as interconnects in integrated circuit (IC) devices causes a difference in strength and can influence hillock formation and electron migration and, thus, system reliability. ${ }^{1,2}$ In metal/ceramic multilayer systems, a textured metal layer affects not only the layer properties (strength and ductility) but also the interface properties (orientation relationships and interfacial toughness). Both the metal layer properties and the interface properties are key parameters that control the fracture toughness of the multilayer. $^{3}$

Energetic ion bombardment during film deposition has been found to have a pronounced effect on both the fiber texture and the in-plane texture, ${ }^{4}$ and there are several models to explain the development of texture in ion-beamassisted deposition (IBAD) films. Dobrev ${ }^{5}$ and Van Wyk and Smith $^{6}$ both suggested that grains with easy channeling direction aligned with the ion beam will remain coolest in the thermal spike, serving as "seeds" for recrystallization of the

a)Electronic mail: hongji@umich.edu surrounding matrix. This model is based on experiments at high ion energy (tens to hundreds of $\mathrm{keV}$ ) and assumes that ion-beam-induced grain growth occurs during deposition. As a result, equiaxied grains with larger grain size form in the films, accompanied by a smooth surface. Bradley et al., ${ }^{7,8}$ on the other hand, developed a model to explain the development of preferred orientation due to low-energy ion bombardment during film growth. This model is based on the fact that different crystallographic orientations have different sputter yields. In a polycrystalline film, grain orientations with higher sputter yields are removed faster by sputtering. The newly deposited layer grows epitaxially on grains with low sputter yield orientation, and these grains will eventually dominate the film. Low-energy ion bombardment usually causes a rough surface due to the difference in sputter rates for different grain orientations. It is obvious from both models that the microstructure and surface morphology of the films will also change as the crystallographic texture is developed. In applications specific to microelectronics and multilayer structural systems described earlier, texture, microstructure, and surface morphology are very important factors in the overall performance of the films. However, little work has been done to relate texture development to microstructure and surface morphology in films deposited by IBAD. 


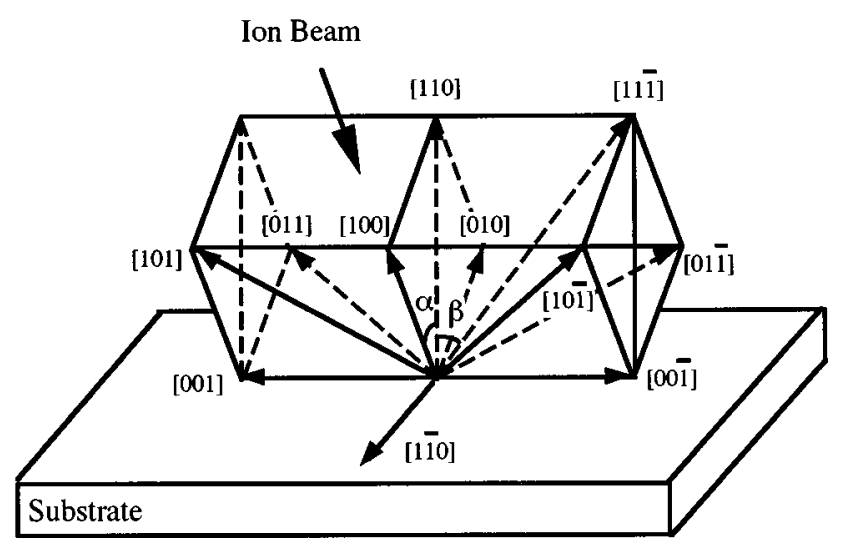

$\alpha=45^{\circ}, \beta=35.26^{\circ}$

(a)

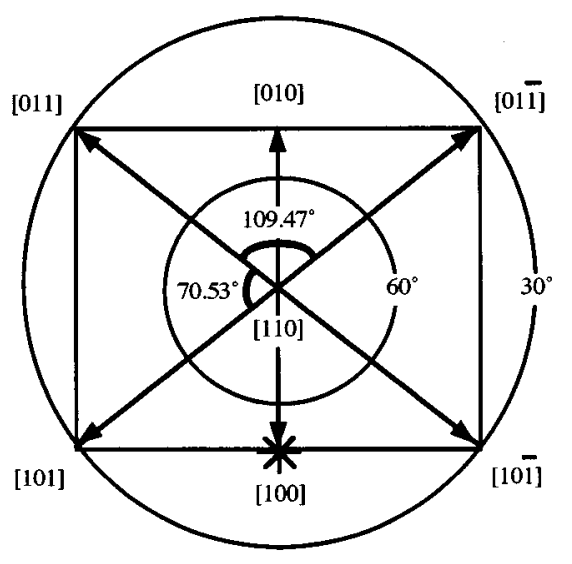

先 Ion Beam Direction

(b)

FIG. 1. Schematics of (a) crystallographic structure of niobium film with $\{110\}$ fiber texture and $\langle 100\rangle$ parallel to ion-beam direction, and (b) pole structure of niobium films with strong in-plane texture as modified by ion bombardment during film deposition.

The purpose of this work is to control the in-plane texture in the niobium films by ion bombardment during film deposition, to examine the effect of normalized energy of the ion beam and film thickness on the degree of texture, microstructure, and surface morphology of the films, and to understand the mechanism that controls the development of both the texture and surface morphology of thin films under ion bombardment. Following is the approach for controlling inplane texture in niobium films.

For bcc films like niobium, a $\{110\}$ fiber texture is generally observed with the close-packed planes parallel to the surface of the substrate to minimize the surface energy. As illustrated in Fig. 1(a), with $\langle 110\rangle$ parallel to the surface nor$\mathrm{mal}$, the two easiest channeling directions $\langle 111\rangle$ and $\langle 100\rangle$ make angles of $35.26^{\circ}$ and $45^{\circ}$ with the surface normal, respectively. In the case of a $45^{\circ}$ incident ion beam, grains with $\langle 100\rangle$ channeling direction aligned with the ion beam will dominate the film, and therefore, the $\langle 110\rangle$ in-plane direction is expected to be parallel to the ion-beam projection on the substrate. An ion-beam incident angle of $35.26^{\circ}$, on the other hand, will favor the growth of the grains with $\langle 111\rangle$ direction aligned along the ion beam, so that the $\langle 100\rangle$ inplane direction is parallel to the projection of the ion beam on the substrate. In this work, we chose an ion-beam incident angle of $45^{\circ}$ to produce an in-plane texture with the $\langle 110\rangle$ direction parallel to the ion-beam projection on the substrate. However, the tilt angles from film surface normal $(\langle 110\rangle$ direction) for the $\langle 100\rangle$ and $\langle 111\rangle$ channeling are $9.74^{\circ}$ apart, and the ion-beam source has a divergence of about $10^{\circ}$. Therefore, an ion-beam incident angle of $50^{\circ}$ was chosen to avoid the mixed effect from $\langle 100\rangle$ and $\langle 111\rangle$ channeling.

As illustrated in Fig. 1(b), films with strong in-plane texture modified by ion bombardment at $50^{\circ}$ should have the following features in their $\{110\}$ and $\{200\}$ pole figures: (i) for the $\{110\}$ pole figure, in addition to the center pole (fiber texture), there are four poles at a tilt angle $\phi=60^{\circ}$ (on the $30^{\circ}$ ring) and the azimuthal angles between them are $70.53^{\circ}$ and $109.47^{\circ}$, respectively; (ii) the $\{200\}$ pole figure has two poles with a tilt angle $\phi=45^{\circ}$ (on the $45^{\circ}$ ring) that bisect the $109.47^{\circ}$ azimuthal angles on the $\{110\}$ pole figures. On the contrary, an absence of in-plane texture in films with $\{110\}$ fiber texture results in (i) a $\{110\}$ pole figure with an intensity maximum at the center and a second intensity maximum on the $30^{\circ}$ ring (tilt angle $\phi=60^{\circ}$ ) with uniform azimuthal intensity; and (ii) a $\{200\}$ pole figure with uniform azimuthal intensity on the $45^{\circ}$ ring. Here, the $\{200\}$ pole figure is used instead of $\{100\}$ pole figure because the structure factor of the $\{100\}$ diffraction for a bcc crystal is zero.

\section{EXPERIMENT}

Niobium films were synthesized in an ultrahigh vacuum (UHV) chamber by vapor deposition with simultaneous ion bombardment (IBAD). The system (Fig. 2) consists of two 6 $\mathrm{kw}$ electron gun evaporating sources with a $15 \mathrm{cc}$ watercooled copper hearth. The electron beam has steering and sweeping capability to control the e-beam coverage on the charge and, hence, the uniformity of the vapor source. Two Cryo-Torr $8^{\circ}$ cryopumps provided a base pressure of $2 \times 10^{-10}$ Torr prior to deposition. An ion gauge and a residual gas analyzer (RGA) were used to monitor the total pressure in the chamber as well as the partial pressure of various components. Argon ions were used in all IBAD depositions. The ion beam was provided by a $3 \mathrm{~cm}$ Kaufman source with ion energy up to $1000 \mathrm{eV}$. The angle between the ion flux and the vapor flux was fixed at $45^{\circ}$. Argon gas flow was typically around $1.50 \mathrm{sccm}$, which corresponded to an argon partial pressure of $1 \times 10^{5}$ Torr in the chamber, controlled by a gas flow controller. The partial pressure of other components such as air, oxygen, and water were all in the low $10^{-10}$ Torr range during deposition, which ensured low contamination in the films. The deposition rates of the two vapor sources were monitored and controlled by two quartz crystal thickness monitors located directly above the hearths, respectively. The ion flux was measured by a Faraday cup attached to the end of the copper block sample 


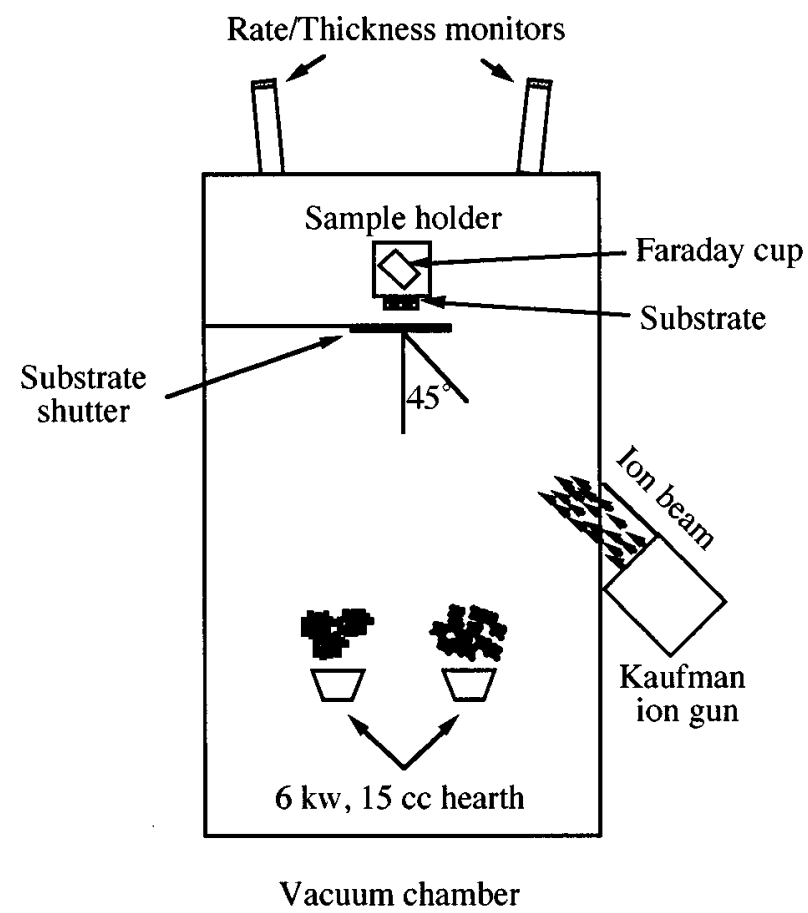

FIG. 2. Schematic of the UHV system used for IBAD.

holder. The angle between the substrate surface normal and the ion beam, hereafter referred as the ion-beam incident angle, was varied by rotating the sample block. A load lock pumped by a turbopump and a mechanical pump is separated from the main chamber by a gate valve, allowing sample interchange without breaking the vacuum in the main chamber.

Depositions were made onto (100) silicon, (0001) sapphire, and amorphous glass. The substrates were cleaned with acetone and methanol prior to introduction to the chamber. Samples used for the texture study were also sputter cleaned with a $500 \mathrm{eV} \mathrm{Ar}^{+}$ion beam at a current of $160 \mu \mathrm{A} / \mathrm{cm}^{2}$ for 6 min prior to deposition. The deposition rate for all experiments was kept close to $0.5 \mathrm{~nm} / \mathrm{s}$. Except for one sample that was deposited without ion bombardment (PVD), all samples were bombarded with an $\mathrm{Ar}^{+}$ion beam with energy between 250 and $1000 \mathrm{eV}$ and ion flux between 4.83 and $193.0 \mu \mathrm{A} / \mathrm{cm}^{2}$. The ion-beam incident angle was $50^{\circ}$ from the substrate surface normal. All depositions were performed at ambient substrate temperature. Niobium films on different substrates with the same deposition conditions (ion energy and $R$ ratio) were deposited at the same time.
Thickness was monitored and controlled by quartz crystal thickness monitors during the deposition, and measured using a Dektak3 profilometer. The thickness for all the samples ranged from 50 to $500 \mathrm{~nm}$. Rutherford backscattering spectrometry (RBS) was used to determine the composition of the films. No impurity other than argon, incorporated from the ion beam, was observed by RBS. The argon level was zero in the PVD film and increased with ion-beam energy and $R$ ratio to a maximum of 5-6 at. \% for the IBAD film deposited at an ion energy of $1000 \mathrm{eV}$ and a $R$ ratio of 0.4 . The oxygen content of the films was measured by nuclear reaction analysis (NRA) using the ${ }^{16} \mathrm{O}(d, p){ }^{17} \mathrm{O}$ reaction with a detection limit of 700 at. ppm. The oxygen level in all the films was less than 1 at. \%.

Morphological features of the as-deposited surface and cross section of the niobium films deposited on (100) silicon substrates were examined by a JOEL JSM-6400 FV fieldemission scanning electron microscope (FESEM) operated at an accelerating voltage of $4 \mathrm{kV}$. Cross sections were obtained by cleaving the niobium films on silicon substrates along the ion-beam direction so that the cleaved surface is parallel to the ion beam. Surface roughness was measured on films deposited on (0001) sapphire substrates by atomic force microscope (AFM) in tapping mode using a Digital Instrument NanoScope III. The crystallographic texture of the niobium films on both (100) silicon and amorphous glass was characterized by Schulz's x-ray pole figure method. Using $\mathrm{Cu} K \alpha$ radiation, the intensities of the $\{110\}$ and $\{200\}$ Bragg diffraction peaks were measured at Bragg angles $2 \theta$ $=38.2^{\circ}$ and $2 \theta=55.1^{\circ}$, respectively, over a range of tilt angle, $\phi$, from substrate normal to $70^{\circ}$ from normal. The data were taken over the full range of azimuthal angle, $\alpha$, from $0^{\circ}$ to $360^{\circ}$.

\section{RESULTS}

\section{A. In-plane texture}

Table I summarizes the deposition conditions for the samples used for texture study. Figures 3 and 4 show the $\{110\}$ and $\{200\}$ pole figures for niobium films deposited on (100) silicon substrates under two different conditions: PVD (Fig. 3), and IBAD at $E=1000 \mathrm{eV}, R=0.4$ (Fig. 4). It should be noted that the ion-beam incident direction was at a tilt angle of $50^{\circ}$ from substrate surface normal and an azimuthal angle of $0^{\circ}$.

Both PVD and IBAD samples have intensity maxima at the center of the $\{110\}$ pole figures. Additional maxima appear on the $30^{\circ}$ ring as indicated in Figs. 3 and 4. For the

TABLE I. IBAD deposition conditions of various samples used for texture study.

\begin{tabular}{cccccc}
\hline $\begin{array}{c}\text { Sample } \\
\text { ID }\end{array}$ & Substrate & $\begin{array}{c}\text { Beam energy } \\
(\mathrm{eV})\end{array}$ & $R$ ratio & $\begin{array}{c}\text { Normalized } \\
\text { energy (eV/atom) }\end{array}$ & $\begin{array}{c}\text { Film thickness } \\
(\mathrm{nm})\end{array}$ \\
\hline 1 & (100) Silicon & 0 & 0 & 0 & 490 \\
2 & (100) Silicon & 250 & 0.1 & 25 & 691 \\
3 & (100) Silicon & 500 & 0.1 & 50 & 737 \\
4 & (100) Silicon & 500 & 0.4 & 200 & 562 \\
5 & (100) Silicon & 1000 & 0.4 & 400 & 828 \\
6 & Glass & 1000 & 0.4 & 400 & 828 \\
\hline \hline
\end{tabular}




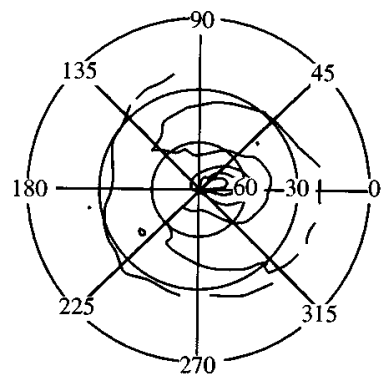

$\{110\}$

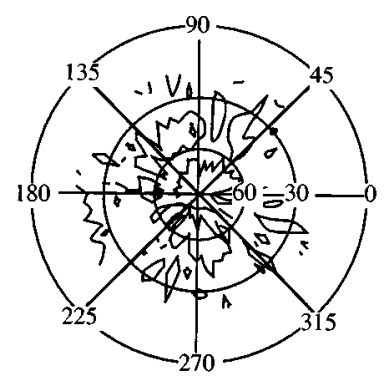

$\{200\}$
FIG. 3. $\{110\}$ and $\{200\}$ pole figures for PVD niobium film deposited on (100) silicon substrate.

PVD sample, the intensity on this $30^{\circ}$ ring is azimuthally uniform. The IBAD sample has four distinct intensity maxima at preferred azimuthal directions on the $30^{\circ}$ ring of the $\{110\}$ pole figure. The azimuthal angles of these four intensity maxima are $47^{\circ}, 117.5^{\circ}, 227^{\circ}$, and $297.5^{\circ}$, respectively, corresponding to two different azimuthal angles separating the four intensity maxima, $70.5^{\circ}$ and $109.5^{\circ}$.

The $\{200\}$ pole figure of the PVD sample has an intensity maximum on the $45^{\circ}$ ring. For the IBAD film, two intensity maxima, identified as $\{200\}$ poles, occur on the $45^{\circ}$ ring at azimuthal angles of $-7.5^{\circ}$ and $172.5^{\circ}$. These two maxima also bisect the two larger angles $\left(109.5^{\circ}\right)$ between the $\{110\}$ poles in the $\{110\}$ pole figure. The results for IBAD niobium film deposited on amorphous glass substrate are the same as for the (100) Si substrate, Fig. 5.

To measure the degree of in-plane texture, we introduced a quantity $D$ (intensity ratio):

$$
D \equiv I_{\text {avg }}^{30^{\circ}} / I^{0^{\circ}},
$$

where $I_{\text {avg }}^{30^{\circ}}$ is the average intensity of the four $\{110\}$ poles on the $30^{\circ}$ ring and $I^{0^{\circ}}$ is the intensity of the center $\{100\}$ pole. Figure 6 shows that the intensity ratio increased linearly with the normalized energy of the ion beam.

\section{B. Surface morphology and microstructure}

The surface morphology of the as-deposited niobium films varies with both the normalized energy of the ion beam

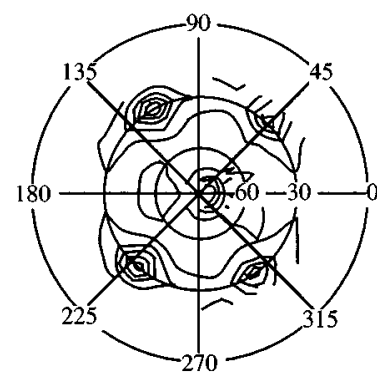

$\{110\}$

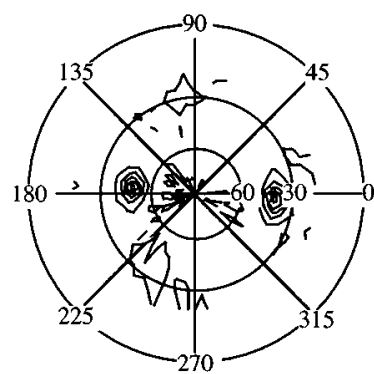

$\{200\}$
FIG. 4. $\{110\}$ and $\{200\}$ pole figures for IBAD niobium film deposited at $1000 \mathrm{eV}, R=0.4$ on (100) silicon substrate.

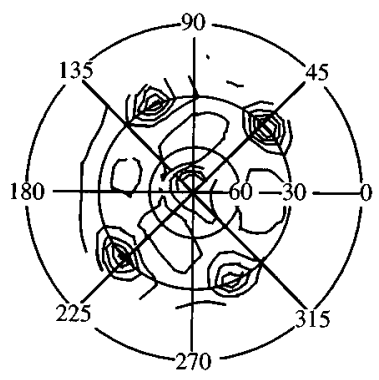

$\{110\}$

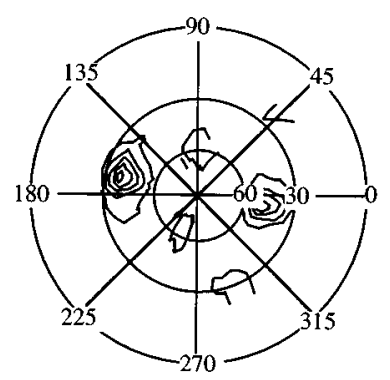

$\{200\}$
FIG. 5. $\{110\}$ and $\{200\}$ pole figures for IBAD niobium film deposited at $1000 \mathrm{eV}, R=0.4$ on amorphous glass substrate.

and film thickness. Figure 7 shows that all films have domelike surface features of increasing dimension with the normalized energy and film thickness. The size of these features ranges from $50 \mathrm{~nm}$ for the IBAD film deposited at $500 \mathrm{eV}$, $R=0.1$ with a nominal thickness of $100 \mathrm{~nm}$, to $400 \mathrm{~nm}$ for the film deposited at $1000 \mathrm{eV}, R=0.4$ with a nominal thickness of $500 \mathrm{~nm}$. These features are elongated with a common orientation, which corresponds to the ion-beam incident direction. It should be noted that for the $1000 \mathrm{eV}, R=0.4$ sample, smaller structures are observed within each feature.

The cross-section micrographs of Fig. 8 reveal a columnar structure for all the films deposited at the three deposition conditions examined. Films deposited at higher ion energy and $R$ ratio have larger columns and more porous structures. The $500 \mathrm{eV}, R=0.1 \mathrm{IBAD}$ film has the finest columnar structure. The IBAD film deposited at $1000 \mathrm{eV}$, $R=0.4$ has the columnar structure tilted toward the ion-beam incident direction. The angle between the columns and the substrate surface normal is about $30^{\circ}$.

The rms surface roughness measured by AFM is presented as a function of the normalized energy $E_{n}$ [Fig. 9(a)], film thickness $t$ [Fig. 9(b)], and the product of the two, $E_{n} \times t$ [Fig. 9(c)]. The PVD film with a nominal thickness of $100 \mathrm{~nm}$ has the smoothest surface with a rms roughness of $1.6 \mathrm{~nm}$. For the IBAD films, the rms roughness increases

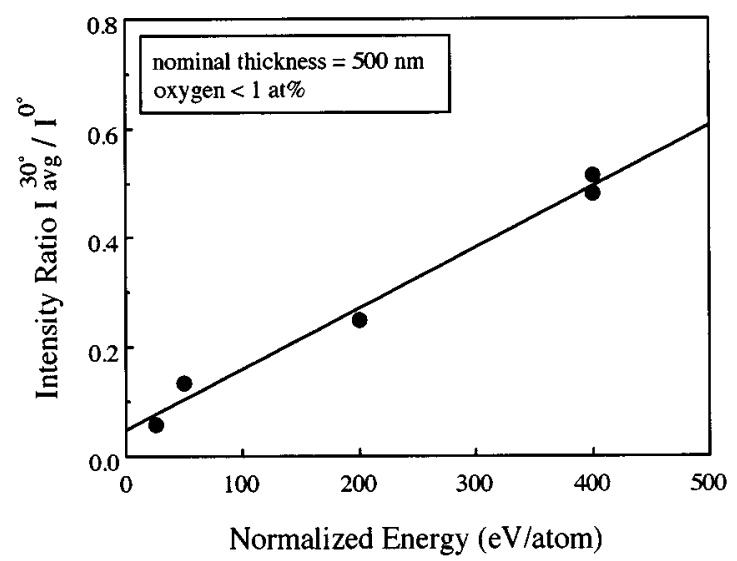

FIG. 6. Intensity ratio $I_{\text {avg }}^{30^{\circ}} / I^{0^{\circ}}$ from the $\{110\}$ pole figures vs normalized energy, indicating an increase in degree of texture in niobium films deposited on (100) silicon substrates as increasing $\mathrm{Ar}^{+}$ion bombardment. 


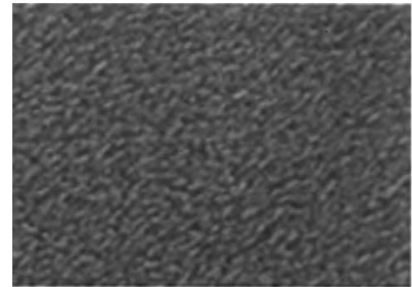

(a) $500 \mathrm{eV}, \mathrm{R}=0.1,500 \mathrm{~nm}$

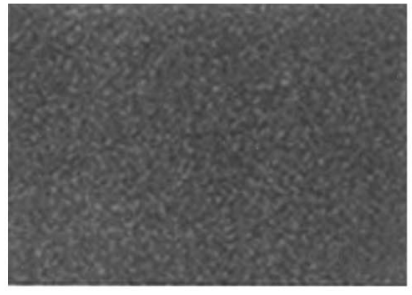

(d) $500 \mathrm{eV}, \mathrm{R}=0.1,100 \mathrm{~nm}$

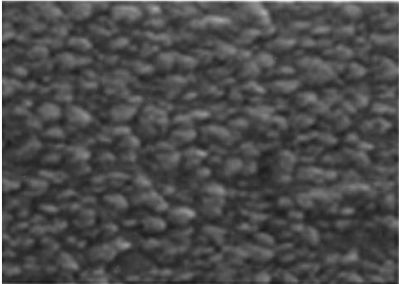

(b) $500 \mathrm{eV}, \mathrm{R}=0.4,500 \mathrm{~nm}$

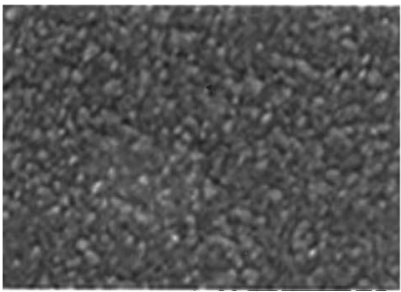

(e) $500 \mathrm{eV}, \mathrm{R}=0.4,100 \mathrm{~nm}$

$00 \mathrm{~nm}$

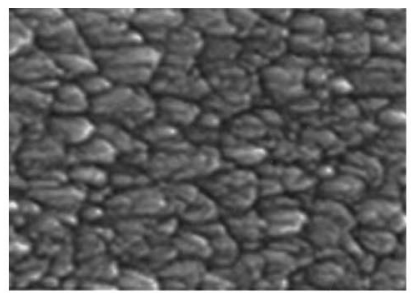

(c) $1000 \mathrm{eV}, \mathrm{R}=0.4,500 \mathrm{~nm}$

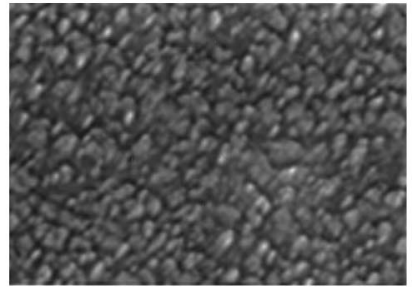

(f) $1000 \mathrm{eV}, \mathrm{R}=0.4,100 \mathrm{~nm}$

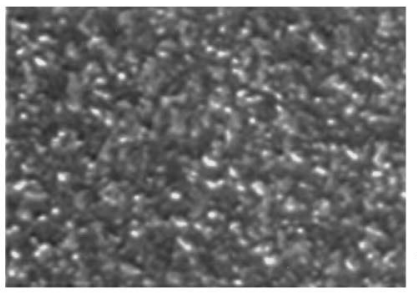

(g) $1000 \mathrm{eV}, \mathrm{R}=0.4,50 \mathrm{~nm}$

FIG. 7. Micrographs showing surface morphology of IBAD niobium films with different thicknesses deposited at different ion energies and $R$ ratios on (0001) sapphire substrates: (a) $500 \mathrm{eV}, R=0.1,500 \mathrm{~nm}$, (b) $500 \mathrm{eV}, R=0.4,500 \mathrm{~nm}$, (c) $1000 \mathrm{eV}, R=0.4,500 \mathrm{~nm}$, (d) $500 \mathrm{eV}, R=0.1,100 \mathrm{~nm}$, (e) $500 \mathrm{eV}$, $R=0.4,100 \mathrm{~nm}$, (f) $1000 \mathrm{eV}, R=0.4,100 \mathrm{~nm}$, and (g) $1000 \mathrm{eV}, R=0.4,50 \mathrm{~nm}$.

with both the normalized energy and film thickness. The thickest film (nominal $t=800 \mathrm{~nm}$ ) deposited at the highest normalized energy $\left(E_{0}=400 \mathrm{eV} /\right.$ atom $)$ has the roughest surface, rms roughness $=36.7 \mathrm{~nm}$. A linear relationship is also found between the rms roughness of the as-deposited surfaces and the product of the normalized energy and the film thickness, $E_{n} \times t$.

\section{DISCUSSION}

\section{A. Texture}

The development of an in-plane texture in niobium films using ion bombardment during deposition was achieved without altering the fiber texture. The pole figure data for PVD and IBAD films reveal that all samples have $\{110\}$ fiber texture, which is thermodynamically favored in vapor deposited bcc metals. The azimuthally uniform intensity of both $\{110\}$ and $\{200\}$ pole figures of the PVD sample indicate the absence of in-plane texture in the PVD film. On the other hand, as expected, both $\{110\}$ and $\{200\}$ pole figures for the IBAD sample show strong in-plane texture in the films as noted by the distinct intensity maxima at preferred azimuthal directions. The angular relationship of these poles match with the crystallographic structure of niobium. Furthermore, the $\{200\}$ pole is parallel to the ion-beam incident direction, and thus, the $\langle 110\rangle$ in-plane direction is parallel with the projection of the ion beam on the film surface. This in-plane texture is a result of $\langle 100\rangle$ channeling. The same result is also found in niobium films deposited on amorphous glass under the same condition ( $1000 \mathrm{eV}, R=0.40)$, implying that there is no substrate effect. This suggests that the process of producing in-plane texture in the niobium films is mainly controlled by the ion beam, and does not result from epitaxial growth.

In-plane texture control by ion bombardment during deposition has been found in several metal systems. Yu et al. ${ }^{9}$ used $200 \mathrm{eV} \mathrm{Ar}^{+}$ion bombardment at an angle of $20^{\circ}$ during sputter deposition of niobium films and observed a strong correlation between the ion-beam current density and the degree of orientation in the films. They proposed that $45^{\circ}$ was a more efficient incident angle, since it allowed the ion beam to align with one of the easiest channeling directions $\langle 100\rangle$ without disturbing the thermodynamically favored $\{110\}$ fiber texture in the films. Sonnenberg et al. ${ }^{10}$ worked with biaxially aligned cubic zirconia films and used IBAD to show the pronounced effect of ion bombardment on texture modification. As pointed out by both groups, the ion-beam incident angle had to be chosen carefully in order to obtain the desired texture.

The mechanism controlling in-plane texture development in niobium films during ion-beam-assisted deposition is 

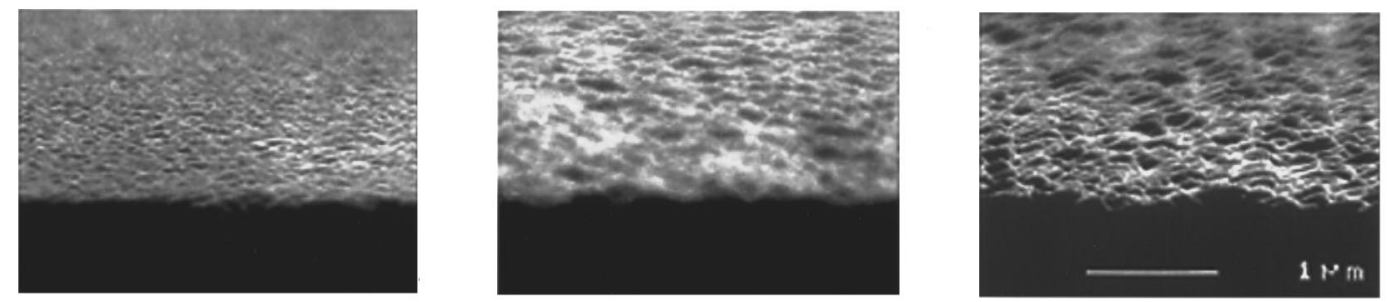

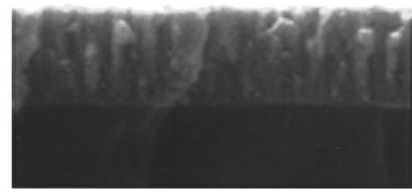

(a) $500 \mathrm{eV}, \mathrm{R}=0.1$

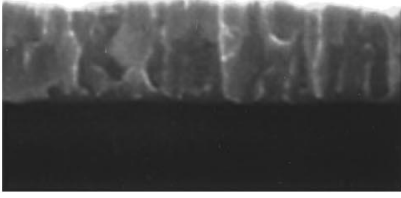

(b) $500 \mathrm{eV}, \mathrm{R}=0.4$

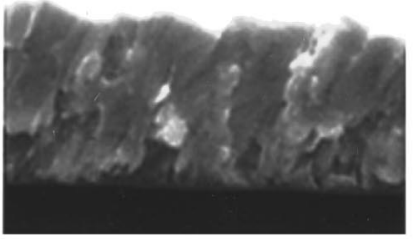

(c) $1000 \mathrm{eV}, \mathrm{R}=0.4$

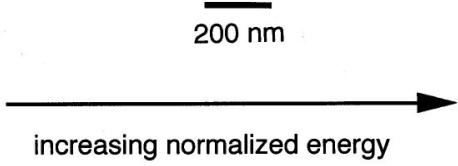

FIG. 8. Micrographs showing surface roughness and fractured cross section of IBAD niobium films deposited on (100) silicon substrates at (a) 500 eV, $R=0.1$, (b) $500 \mathrm{eV}, R=0.4$, and (c) $1000 \mathrm{eV}, R=0.4$.

channeling. When an ion beam channels, the sputter yield is reduced. Hence, sputter rate (sputter yield times ion flux) is low for certain low index crystallographic directions (e.g., $\langle 111\rangle$ and $\langle 100\rangle$ for bcc crystals) due to channeling. ${ }^{11}$ This crystallographic dependence of channeling and sputtering results in a difference in the net growth rate for a particular grain orientation compared to its neighbors. Ion bombardment helps the selective growth of grains that have channeling directions aligned with the ion-beam direction, beginning in the early stage of film growth. Grains having their easy channeling direction aligned with the ion-beam direction (grain A in Fig. 10) grow faster due to the low sputter rate. On the other hand, the growth of misoriented grains (grain $\mathrm{C}$ in Fig. 10) is suppressed because of a high sputter rate. Shadowing by neighboring, faster growing grains augments the difference in growth rate. As a result, grains with either $\langle 100\rangle$ or $\langle 111\rangle$ directions parallel to the ion-beam direction grow preferentially, and eventually dominate the films (Fig. 10). Given the $\{110\}$ fiber texture in the film and the $50^{\circ}$ ionbeam incident angle, in-plane texture developed in the film with the majority of grains having their $\langle 100\rangle$ direction parallel to the ion-beam incident direction.

According to this model of selective growth, ion-beam parameters that affect the sputter rate will also affect the net growth rate, and hence, the degree of texture in films. In the energy range used in this study $(0-1000 \mathrm{eV})$, the sputter yield increases linearly with ion energy, $E .{ }^{11}$ Ion flux is proportional to the $R$ ratio for a given deposition rate. Therefore, an increase in either the ion energy, $E$, or $R$ ratio gives rise to a linearly increasing sputter rate, resulting in a linearly increasing degree of texture. It is not surprising that since the degree of texture increases linearly with the ion energy, $E$ and $R$ ratio, it should also increase with their product, $E \times R=E_{n}$. Since this mechanism for texture development is based on the different sputter rates of different grain orientations due to channeling and the selective growth of certain grains by ion bombardment, no substrate effect is observed.

\section{B. Surface morphology and microstructure}

We observed that the presence of energetic ion bombardment during film growth caused a rougher surface in the niobium films. The rms roughness of these films increases with the normalized energy of the ion beam and film thickness. This observation is in agreement with the work by Erck and Fenske on silver deposited by IBAD on aluminum oxide substrates. ${ }^{12}$ They found that high-energy $(1000 \mathrm{eV})$ and high $R$ ratio $(>0.16)$ ion bombardment caused substantial film porosity and surface roughening while high-energy, but low $R$ ratio or low-energy, ion bombardment produced much smoother surfaces. Similar results were found for other metal coatings produced by IBAD, such as chromium, ${ }^{13}$ niobium, ${ }^{14}$ and nickel. ${ }^{15}$ However, for iron disilicide (Ref. 16) and $\mathrm{Nb}-\mathrm{Cr}$ alloy (Ref. 17) films deposited by IBAD, a drastic reduction in surface roughness was observed with respect to those grown by standard deposition techniques. The smoother surface of the IBAD $\mathrm{Nb}-\mathrm{Cr}$ alloy film was believed to be caused by the enhanced formation of a finely structured intermetallic phase under ion bombardment.

The microstructure of niobium films is also affected by ion bombardment during film deposition. As shown in Fig. 8, the niobium films with ion bombardment at $1000 \mathrm{eV}$, 


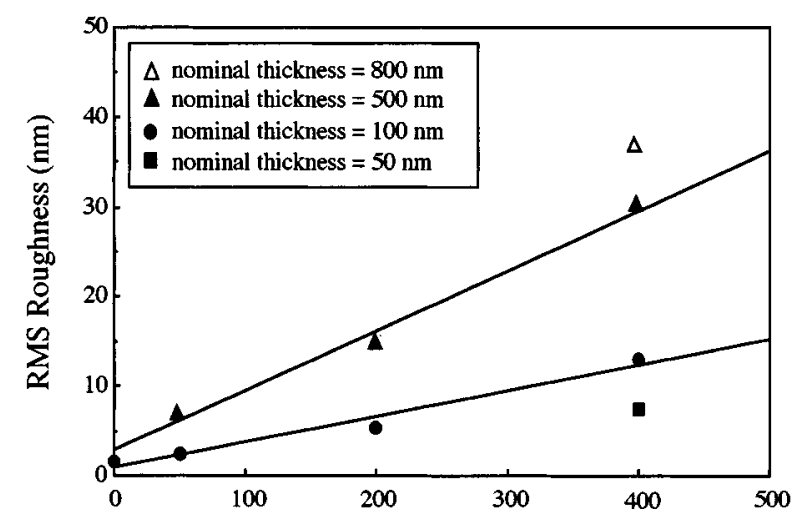

(a)

Normalized Energy (eV/atom)

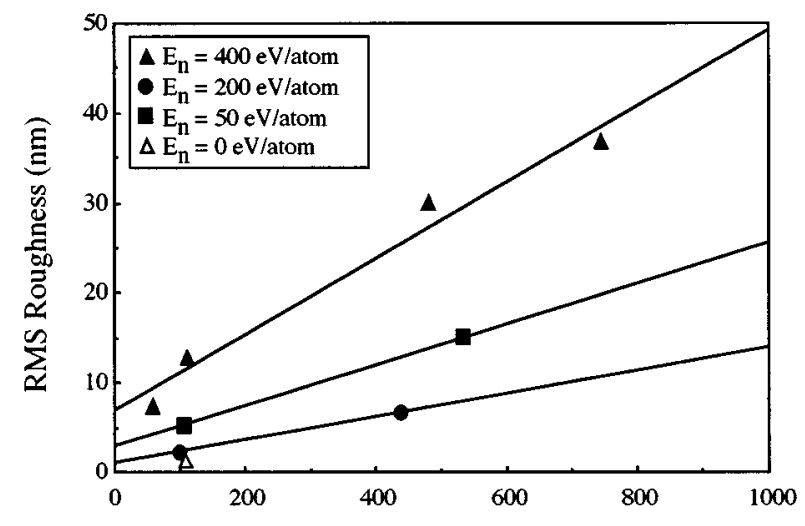

(b)

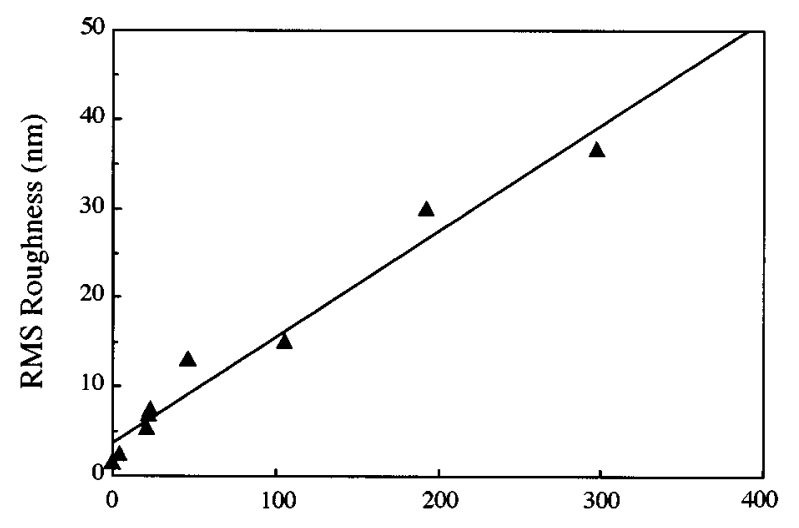

(c) Normalized Energy x Film Thickness ( $\mu \mathrm{m}-\mathrm{eV} /$ atom)

FIG. 9. The rms roughness of as-deposited surface of niobium films deposited on (100) silicon substrates as a function of (a) normalized energy, (b) film thickness, and (c) the product of the two.

$R=0.4 ; 500 \mathrm{eV}, R=0.4$; and $500 \mathrm{eV}, R=0.1$ all have columnar structures. The width of the columns increases with the normalized energy of the ion beam. The columns for the $1000 \mathrm{eV}, R=0.4$ film are tilted towards the ion-beam incident direction at $30^{\circ}$, although the ion beam was incident at $50^{\circ}$. This result follows the empirical relationship between the column orientation and the deposition angle in PVD depositions for a deposition angle less than $60^{\circ}$ :

$$
\tan \alpha=2 \tan \beta,
$$

widely known as the "tangent law,",18 where $\alpha$ is the depo-

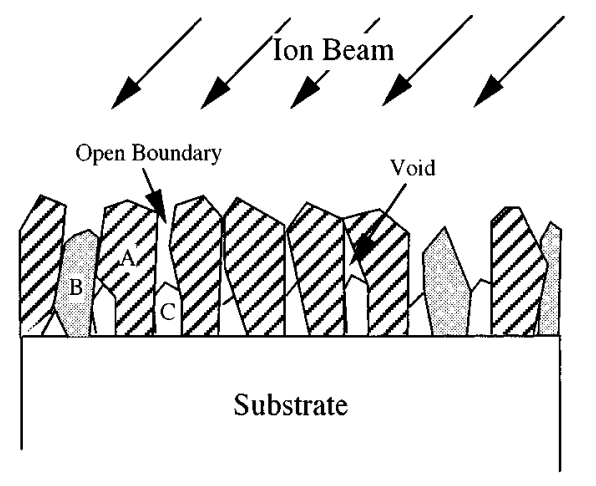

FIG. 10. Schematic illustrating the texture development and microstructure and surface morphology evolution in thin films under ion bombardment during film deposition.

sition angle and $\beta$ is the column orientation where both angles are relative to the substrate normal. It is also in good agreement with the molecular dynamics simulation of oblique deposition by Srolovitz et al. ${ }^{19}$ who used an energetic vapor flux instead of the ion beam and found that the relationship between $\alpha$ and $\beta$ fit the "tangent law."

Voids and porosity are also observed in the niobium films. The films deposited at $1000 \mathrm{eV}, R=0.4$ have the most porous structure and open boundaries. This is a common observation for metal films deposited by IBAD. Srolovitz et $a l .{ }^{19}$ observed that the elongated voids and porous microstructure formation in their molecular dynamics simulation are dependent on the deposition kinetic energy and substrate temperature. Hsieh et al. studied the microstructure of niobium films deposited on 316LSS substrates. ${ }^{14}$ They found the finest structure in the IBAD film with medium energy ion bombardment. IBAD films with both low-energy and low $R$ ratio, and high-energy and high $R$ ratio ion bombardment produced structures containing much porosity. Although the formation of a porous structure at low energy and low $R$ ratio can be explained by the film densification mechanism, the understanding of the formation of such a structure at highenergy and high $R$ ratio ion bombardment is not as straightforward. Both Erck and Hsieh attributed the rough surface and porous structure of films with ion bombardment at high normalized energy to the cascade damage caused deeper in the films and the formation of depleted zones where the deposited vapor could not reach.

We suggest that the mechanism controlling the in-plane texture development described in the previous section is also responsible for the observed surface morphology and microstructure of the IBAD films. Different grain orientations have different sputter rates under ion bombardment due to channeling, and thus, have different net growth rates. As shown in Fig. 10, the growth of grains having a higher sputter rate (grain C) is suppressed under ion bombardment at high normalized energy. Meanwhile, grains with their channeling directions aligned with the ion-beam direction (grain A) grow preferentially due to the smaller sputter rate. Grains with higher net growth rate grow higher, receive the arriving vapor flux, and shadow their neighboring grains. In some cases, they coalesce with one another forming voids in the structure, and in other cases they leave open boundaries in the 
films. A rough as-deposited surface forms as a result of the different heights among grains due to the difference in net growth rate. Surface diffusion, which is important in the growth process, when the deposition temperature is above one-third of the melting temperature of the metal, is very limited in this case. Given the fact that the depositions were near ambient temperature $\left(\sim 100^{\circ} \mathrm{C}\right)$, and that niobium has a high melting temperature $\left(2468{ }^{\circ} \mathrm{C}\right)$, it is understandable that even the enhanced adatom mobility introduced by ion bombardment is still insufficient for surface diffusion to affect the surface morphology.

The dependence of film surface roughness on the ionbeam normalized energy and the film thickness can be understood from the following analysis: Suppose that vapor deposition occurs at a rate $r$, sputtering for grain $\mathrm{A}$ at a rate $s_{A}$, and for a neighboring grain B (Fig. 10) with a different orientation at a rate $s_{B}$, and the total deposition time is $t$. The height difference between grains $\mathrm{A}$ and $\mathrm{B}, \Delta h$, can be written as

$$
\Delta h=\left(r-s_{A}\right) \times t-\left(r-s_{B}\right) \times t=\left(s_{B}-s_{A}\right) \times t,
$$

where $s_{A, B}=Y_{A, B} \times I . \quad Y_{A, B}$ is the sputter yield for grains A and $\mathrm{B}$, respectively, and $I$ is the ion flux. For the energy range used in this work $(0-1000 \mathrm{eV})$, the sputter yield is a linear function of ion energy $E,{ }^{11}$ so $Y_{A, B}=C_{A, B} \times E$ and $C_{A, B}$ is a constant for grains $\mathrm{A}$ and $\mathrm{B}$, respectively, giving

$$
\begin{aligned}
\Delta h & =\left(C_{B}-C_{A}\right) \times E \times I \times t \\
& =\left(C_{B}-C_{A}\right) \times E \times R \times r \times t \\
& =\left(C_{B}-C_{A}\right) \times E_{n} \times h_{n},
\end{aligned}
$$

where $R$ is the ion-to-atom arrival rate ratio and $h_{n}=r \times t$ is the nominal height of the film. With the assumption that the major contribution of surface roughness comes from the difference in the height of adjacent grains, it is evident from Eq. (4) that the surface roughness of the as-deposited film is linearly proportional to the normalized energy $E_{n}$, and the film thickness $h_{n}$. Thus, the surface roughness of the asdeposited films is controlled by the same deposition parameters (ion energy and $R$ ratio) that control the in-plane texture development in the films.

\section{CONCLUSION}

Ion bombardment during film deposition has a pronounced effect on the in-plane texture, the microstructure, and the surface morphology of niobium films. While a $\{110\}$ fiber texture is thermodynamically favored for vapor deposited niobium (bcc) films, a strong in-plane texture is developed by selected angle ion bombardment. With an ion-beam incident angle of $50^{\circ}$, the niobium film texture has azimuthal orientation with $\langle 110\rangle$ direction parallel to the projection of the ion beam onto the substrate surface. The degree of the texture is a strong linear function of the product of the ion energy, $E$, and $R$ ratio. All films studied have a columnar structure. Domelike surface features oriented along the ionbeam incident direction are observed on all as-deposited sur- faces of the films. The dimension of these features correlated well with the rms roughness of the surfaces, which increases linearly with the normalized energy, and the film thickness. The rms roughness is $1.6 \mathrm{~nm}$ for the PVD film with a nominal thickness of $100 \mathrm{~nm}$ and increases to $36.7 \mathrm{~nm}$ for the 800 $\mathrm{nm}$ thick IBAD film with normalized energy $E_{n}$ $=400 \mathrm{eV} /$ atom.

The mechanism controlling both the texture development and the microstructure and surface morphology modification is differential sputtering caused by ion channeling. When the ion beam is aligned with one of the easy channeling directions in a grain, the sputter yield and, thus, the sputter rate, are reduced, resulting in preferential growth of this grain. The results of the in-plane texture development and surface morphology modification are a combination of the preferential growth and shadowing effect. This work shows that both film integrity and texture development can be controlled by optimizing the ion bombardment conditions.

\section{ACKNOWLEDGMENTS}

The authors acknowledge Christopher Rood at Sandia National Laboratories at Livermore for help with the fieldemission scanning electron microscope. The authors would also like to thank the Michigan Ion Beam Laboratory for Surface Modification and Analysis and the Electron Microbeam Analysis Laboratory at the University of Michigan for the use of their facilities. This work is supported under NSF Grant No. DMR-9411141. One of the authors (N.R.M.) gratefully acknowledges the support of the U.S. DOE through Contract No. DE-AC04-94AL85000.

${ }^{1}$ J. E. Sanchez, Jr., Mater. Res. Soc. Symp. Proc. 343, 641 (1994).

${ }^{2}$ P. R. Besser, J. E. Sanchez, Jr., S. Brennan, J. C. Bravman, G. Takaoka, and I. Yamada, Mater. Res. Soc. Symp. Proc. 343, 659 (1994).

${ }^{3}$ G. S. Was and T. Foecke, Thin Solid Films 286 (1996).

${ }^{4}$ F. A. Smidt, Int. Mater. Rev. 35(2), 61 (1990).

${ }^{5}$ D. Dorbev, Thin Solid Films 92, 41 (1982).

${ }^{6}$ G. N. Van Wyk and H. J. Smith, Nucl. Instrum. Methods 170, 433 (1980).

${ }^{7}$ R. M. Bradley, J. M. Harper, and D. A. Smith, J. Appl. Phys. 60, 4160 (1986).

${ }^{8}$ R. M. Bradley, J. M. Harper, and D. A. Smith, J. Vac. Sci. Technol. A 5, 1792 (1987)

${ }^{9}$ L. S. Yu, J. M. E. Harper, J. J. Cuomo, and D. A. Smith, J. Vac. Sci. Technol. A 4, 443 (1986).

${ }^{10}$ N. Sonnenberg, A. S. Longo, M. J. Cima, B. P. Chang, K. G. Ressler, P. C. McIntyre, and Y. P. Liu, J. Appl. Phys. 74, 1027 (1993).

${ }^{11}$ H. S. Roosendaal, in Sputtering by Particle Bombardment I-Physical Sputtering of Single-Element Solids, edited by R. Behrisch (Springer, New York, 1981), p. 219-256.

${ }^{12}$ R. A. Erck and G. R. Fenske, Thin Solid Films 181, 521 (1989).

${ }^{13}$ C. C. Cheng, R. A. Erck, and G. R. Fenske, Mater. Res. Soc. Symp. Proc. 140, 177 (1989).

${ }^{14}$ J. Hsieh, R. Lee, R. A. Erck, G. R. Fenske, Y. Y. Su, M. Marek, and R. F. Hochman, Surf. Coat. Technol. 49, 8386 (1991).

${ }^{15}$ K. H. Muller, Phys. Rev. B 35, 7906 (1987).

${ }^{16}$ T. Terrasi, S. Ravesi, C. Spinella, M. G. Grimaldi, and A. La Mantia, Thin Solid Films 241, 188 (1994).

${ }^{17}$ J. H. Hsieh, W. Wu, R. A. Erck, G. R. Fenske, Y. Y. Su, and M. Marek, Surf. Coat. Technol. 51, 212 (1992).

${ }^{18}$ J. M. Nieuwenhuizen and H. B. Haanstra, Philips Tech. Rev. 27, 87 (1966).

${ }^{19}$ L. Dong, R. W. Smith, and D. J. Srolovitz, J. Appl. Phys. 80, 5682 (1996). 